\title{
A Coalitional Game Approach for Packet Delivery in Mobile Adhoc Network
}

\author{
S.V.Karthik ${ }^{1,}$ V.Shyamala ${ }^{2}$ \\ ${ }^{1}$ Assistant Professor, Prist University, Kumbakonam, Tamil Nadu, India. \\ ${ }^{2}$ Prist University, Kumbakonam, Tamil Nadu, India.
}

\begin{abstract}
A coalitional game is residential to study the actions of the rational mobile nodes for supportive packet delivery. We consider the problem of supportive packet delivery to mobile nodes in a cross wireless mobile network, where both wired and wireless network communications are used. A group of mobile nodes makes a conclusion to join or to leave a coalition based on their creature payoffs. The individual payoff of each mobile node is a function of the usual delivery delay for packets transmitted to the mobile node from a base station and the cost incur by this mobile node for relay packets to other mobile nodes. We propose a solution based on a coalition formation among mobile nodes to cooperatively deliver packets among these mobile nodes in the similar coalition. To discover the payoff of each mobile node, a continuous time Markov chain model is formulated and the expected cost and packet delivery postponement are obtained when the mobile node is in a coalition. Because both the expected cost and packet delivery delay depend on the possibility that each mobile node will aid other mobile nodes in the same coalition to forward packets to the destination mobile node in the same coalition, a bargaining model game is used to find the best helping probabilities. Behind the payoff of every mobile node is obtained, we discover the resolutions of the coalitional game which are the constant coalitions. A distributed algorithm is reachable to obtain the constant coalitions and a Markov-chain-based analysis is used to estimate the constant coalitional structures obtained from the distributed algorithm. Performance estimate results show that when the constant coalitions are formed, the mobile nodes realize a higher payoff. With a coalition formation, the mobile nodes achieve superior payoff than that when every mobile node acts alone.
\end{abstract}

Keywords: Hybrid wireless network, network social analysis, cooperative packet delivery, coalitional game, bargaining model game, carry-and-forward-based data delivery.

\section{Introduction}

Coalition formation has been a research topic of continuing interest in the area of coalitional games. It has been examined from some points of vision starting with [15], where the static situation of cooperative games in the presence of a given coalition structure (i.e., a partition) was considered. Early research on the subject is discussed in [16]. More recently, the problem of formation of stable coalition structures was considered in [17] in the presence of externalities and in [18] in the presence of binding agreements. In both papers two-stage games are analyzed. In the first stage coalitions form and in the second stage the players engage in a non cooperative game given the emerged coalition structure. In this situation the query of constancy of the coalition formation is then studied. Much research on stable coalition structures focused on hedonic games. In other words, a payoff of a player is a preference relation on the sets of players that include him. [19] considered four forms of stability in such event: interior Nash, individual and contractually creature constancy. Each alternative confines the plan that refusal player, respectively, refusal set of players has an incentive to change the existing coalition structure. In two models (i.e. symmetric and asymmetric) for cooperative resource bargaining model in shared networks based on the concept of preference functions introduced by Cao in [20]. The symmetric model assumes that all players have equal bargaining model powers while in the asymmetric container, players are permitted to present bids to the network operator to control the final bargaining model outcomes. a bargaining model game is used to find the best helping possibility for every part of the mobile nodes in a coalition. The bargaining model solutions proposed vary according to a parameter that considers the tradeoff of one's gain and the sufferers of others.

A few works in the literature proposed communication models for adhoc networks with relay-based schemes [2],[3], [4] to decrease the wait of data relief. In this design, mobile nodes in a cluster kindly send data packets with each other. However, the kind of hypothesis here is that the mobile nodes in the similar group always help each other for data delivery. since a transaction subsists among performance improvement and communication cost for such cooperative data delivery, this assumption may not be always correct. For example, when a mobile node has partial conduction bandwidth and is of self-centeredness, it may not connect a collection for cooperative data delivery. In this situation the hypothesis of coalitional structure [5] can be applied to analyze the dynamics of coalition (or group) formation among mobile nodes. Coalitional events have 
been used to form and examine the resource allotment crisis in wireless networks. In [6], mobile nodes form coalitions and cooperatively share the limited bandwidth of vehicle-to-roadside links to achieve high spectrum utilization. In [7], roadside BSs form coalitions in which the BSs in the same coalition cooperatively coordinate the classes of data that they transmit to mobile nodes, and thereby, develop their revenue.

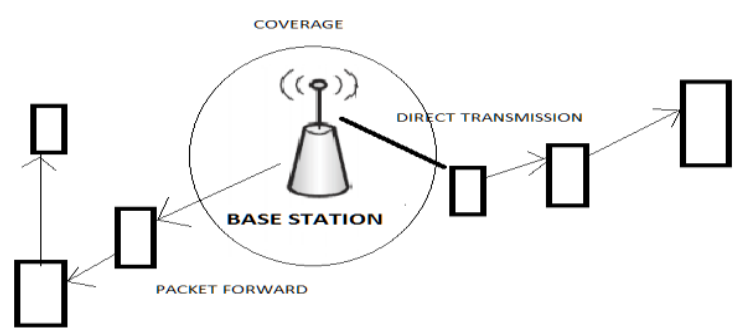

Fig.1. . In a adhoc network the mobile nodes can form coalitions to help forward data from a base station to other mobile nodes which are out of the transmission variety of the base station.

In the scenario under consideration, a base station has packets to send out to a mobile node which may not be in the broadcast collection of the BS. To diminish the wait of packet delivery, coalitions can be formed. The NSA among the mobile nodes can be exploited to decrease the complexity of coalition formation [1]. Mobile nodes in the similar coalition aid each other to deliver packets sent from the BS to the destination mobile nodes. Based on a coalitional game formation, we learn the dynamics of the behavior of mobile nodes helping each other to forward data packets based on their individual selfishness with an objective to maximizing their individual payoffs.

\section{The major assistances of the paper can be summarized as follows:}

We establish a coalitional game formulation to study how mobile nodes can dynamically form coalitions to cooperatively forward data of other mobile nodes in the same coalition. We apply NSA to decrease the computational complexity of coalition formation. Two resolution concept are constant coalitional formation and hub, are measured for the proposed coalitional formation. We propose a bargaining game formulation to attain Pareto-most favorable resolution for the probabilities that mobile nodes will help other mobiles in the same coalition. A distributed coalition formation algorithm is proposed which guarantees that constant coalitional formation can be obtained. We perform a inclusive performance estimation of the proposed method.

\section{System Model}

The proposed framework will be practical for sustaining various mobile applications based on distributed cooperative packet delivery.

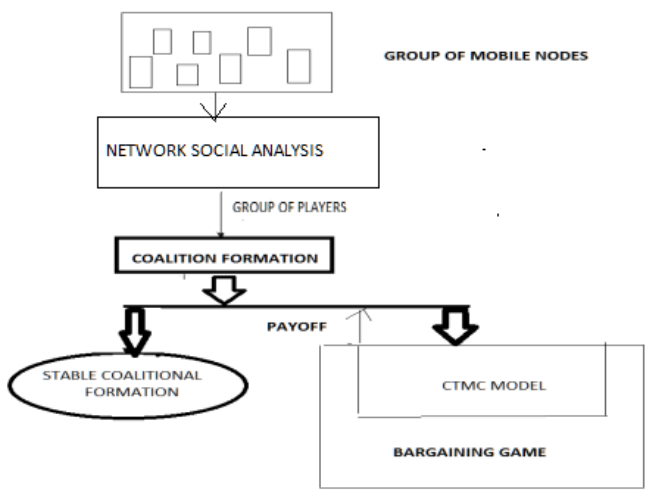

Fig.2. Diagram showing the interrelationship among the three steps, namely, mobile node using NSA, bargaining model game, and coalitional game.

The proposed method consists of three interconnected steps as shown in Fig. 2. We initial use a NSAbased approach [8], [9], [10] to be familiar with which mobile nodes have the potential to aid other mobile nodes for data delivery in the similar group. After the NSA based mobile node grouping is finished, the mobile nodes in each group cooperate a coalitional game to obtain a constant coalitional structure. The payoff of every mobile node is a function of cost incurred by the mobile node in communicating packets and the release wait for 
packets spreaded to this mobile node from a BS. A continuous-time Markov chain (CTMC) model [1] is originated to attain the expected cost and packet delivery delay for each mobile node in the similar coalition. Since the expected cost and packet delivery delay vary with the probability that each mobile node helps other mobile nodes deliver packets, a bargaining model game[11] , is used to find the best helping probabilities for all the mobile nodes. For every mobile node, after the optimal probability of helping other mobile nodes is obtained, we can find out the payoff of every mobile node when it is a member of its current coalition. It obtained from this game are used to determine the solution of the coalitional game in terms of stable coalitional structure. A distributed algorithm is used to attain the solution of the coalitional game and a Markov chain-based analysis is presented to evaluate the stable coalitional structures obtained from the distributed algorithm.

We suppose that the packets are not instantly discarded from the cache of the BSs or the mobile nodes after they are throw or promoted. In addition, there is a controller at the submission server which gather mobility information of the nodes by using the subsequent method:

- When the mobile nodes meet each other, they make a proof of the time they meet.

- Given a definite time period (e.g., 1 hour), the mobile nodes compute the meet rate with other nodes by separating the number of meet by the length of the time period.

- The mobile nodes provide the meet rate in sequence to the controller at the submission server irregularly.

- The controller maintains a record of the meet rate information for all the mobile nodes in the network, and this record is used for network social analysis (NSA). Also, the controller manages the information swap among the base stations or access points.

\section{Stable Coalition Formation For Cooperative Packet Delivery}

The proposed scheme show that when the stable coalitions are formed, the mobile nodes achieve a nonzero payoff .

\section{1 NETWORK SOCIAL ANALYSIS}

In this section, we present a process for mobile node grouping based on social network investigation. The main difficulty of coalition structure is that the computational complexity increases exponentially when the number of nodes increases [5], [12]. that's why, the most important purpose of the anticipated NSA-based mobile node grouping is to decrease the complexity of coalition structure when there are various mobile nodes participating in the supportive information delivery scheme. The key method of the NSA-based mobile node grouping is to sort out several mobile nodes which will not give to the supportive packet delivery(i.e., to split the mobile nodes into multiple social groups in which mobile nodes in a social group do not help with the mobile nodes in another social group). A social network or a group is collected of nodes and ties. In this representation, every mobile node is a node and interaction of mobile nodes are ties. Whether or not a tie will be recognized between two nodes can be resoluted by using centrality metrics used in graph hypothesis and network investigation. Centrality is a quantification of the relative consequence of a highest point within the graph (e.g., how important a node is within a social group). We recognize how every node is important to others based on the Poisson modeling of the network which is called Poisson process based centrality. To recognize groups of mobile nodes using their Poisson process-based centrality, we suggest an algorithm which ensures that for each mobile node in the same group, the possibility that the packet delivery postponement residues below a necessary time interval, can be maintained above a end threshold. Many mobile nodes can assist and appearance coalitions.We presume that every mobile node in the equal coalition will bring and onward packets to other mobile nodes when they gather each other. Each mobile node $k \in I N=\{1 \ldots . N\}$ has a communication range of $g_{k}$ meters. We believe that over a period of time (e.g., 1 hour), we can expect the mobility and interencounter time pattern of every mobile node.Due to the property of speed and density of mobile nodes, the meet-associated statistical data may differ [21]. In such a case, the mobility and interencounter time pattern of mobile nodes composed through a small time period can be uttered as temporary social make contact with pattern which can be more useful than the increasing contact pattern to recover bring-and-onward-based data delivery [22]. Let mobile node $\mathrm{k}$ meet another mobile node $\mathrm{i}$ on the path with rate $r_{\mathrm{ki}}=\mathrm{r}_{\mathrm{ik}}$ per unit of point in time and the number of encounters between mobile node $\mathrm{k}$ and mobile node $\mathrm{i}$ during a period of time is nki= nik. Let $\mathrm{rk} 0$ and $\mathrm{r} 0 \mathrm{k}$ be the rates that mobile node $\mathrm{k}$ assembles the base station and vice versa. Note that " 0 " is used as the index of any base station and its transmission range is g0. The encounter method for every couple of nodes is implicit to pursue a Poisson process and the encounter rate is used as the matching constraint. For the encounter process, that the stochastic properties can be characterized by the Poisson hypothesis, was acceptable in [23], [24]. It was given away that the encounters between a couple of mobile nodes pursue a Poisson distribution if the nodes move in a narrow region. every mobile node $\mathrm{k}$ is preparing to help other mobile nodes to deliver packets with 
probability pk (i.e., $\mathrm{pk}=1$ if mobile node $\mathrm{i}$ always receives information packets, bring, and forwards them to other mobile nodes). Any mobile node k receives packet(s) from a BS or from other mobile node i in the similar coalition at the cost of $c^{r}$ ki per packet. Mobile node $k$ then forwards the packet(s) to its destination or to another mobile node i'in the similar coalition at the cost of $\mathrm{c}_{\mathrm{ki}}^{\mathrm{f}}$, per packet We suppose that every mobile is capable to identify whether the other mobile nodes have the similar packet(s).

To guarantee that mobile node $\mathrm{i}$ will deliver a packet received from the base station to mobile node $\mathrm{k}$ in the essential time $T_{k}$, we believe that if mobile node $\mathrm{i}$ is called by the base station in a time interval of T0i=1/r0i and then contacted by mobile node $\mathrm{k}$ within an interval of Tik $=1 / \mathrm{r} 0 \mathrm{i}$, the probability that the data packet will be delivered from the base station to mobile node $\mathrm{k}$ via mobile node $\mathrm{i}$ is

$$
\begin{aligned}
\mathrm{P}_{\mathrm{ki}}\left(\mathrm{T}_{0 \mathrm{i}}+\mathrm{T}_{\mathrm{ik}}<\mathrm{T}_{\mathrm{k}}\right) & =\int_{0}^{T k} f_{0 \mathrm{k}}(\mathrm{t}) \otimes f_{\mathrm{nk}}(\mathrm{t}) \mathrm{dt} \\
& =\iint_{0}^{t} f_{0 \mathrm{k}}\left(\mathrm{t}^{\prime}\right) \mathrm{f}_{\mathrm{ik}}\left(\mathrm{t}^{-} \mathrm{t}^{\prime}\right) \mathrm{dt} \mathrm{t}^{\prime} \mathrm{dt}
\end{aligned}
$$

Where $\otimes$ is the convolution operator, and f0i(t) and fik $(\mathrm{t})$ for $\mathrm{t} \geq 0$ are the probability density functions (PDFs) of TOi and Tik, respectively. f0i(t) and fik(t) are given by

exponential PDFs. Hence, the PDF of random time interval $t$ that mobile nodes $k$ and $i$ will contact each other is givenby: $\mathrm{fki}(\mathrm{t})=$ rki $e-r k i t$ where rki is the encounter rate between mobile node $\mathrm{k}$ and mobile node $\mathrm{i}$. Note that f0i(t) and fik(t) are general and can be any other PDF rather than the exponential PDF.

Algorithms 1 under recognizes the collection of mobile nodes.

The nodes in such a collection are the players in the bargaining model game and the coalitional game. In this algorithm, IN denotes the location of all mobile nodes and XK is a vector denoting the relationship of mobile node $\mathrm{k}$ with other mobile nodes.

Algorithm 1. NSA-based mobile node grouping algorithm.

1: Exchange profile information (i.e., encounter

information) among mobile nodes. Set $\mathrm{S}=\phi / / \mathrm{a}$

temporary variable.

2: Initialize locate of associations for all mobile nodes, i.e., $\mathrm{X}_{\mathrm{K}}=\phi, \forall_{\mathrm{k}} \in \mathrm{IN}$

3: for each mobile node $\mathrm{k} \in \mathrm{IN}=\{1 \ldots \ldots . \mathrm{N}\}$

4: $\mathrm{S}=\mathrm{S} \cup\{\mathrm{k}\}$

5: for each mobile node $\mathrm{I} \in \mathrm{IN} \backslash \mathrm{S}$

6: if $\left(\mathrm{P}_{\mathrm{ki}}\left(\mathrm{T}_{0 \mathrm{k}}+\mathrm{T}_{\mathrm{ki}}<\mathrm{T}_{\mathrm{i}}\right) \geq \mathrm{w}_{\mathrm{k}}\right.$ and

$\mathrm{P}_{\mathrm{ik}}\left(\mathrm{T}_{0 \mathrm{k}}+\mathrm{T}_{\mathrm{ki}}<\mathrm{T}_{\mathrm{i}}\right) \geq \mathrm{w}_{\mathrm{i}}$ and $\left.\mathrm{n}_{\mathrm{ki}}>\mathrm{n}_{\mathrm{th}}\right)$

7: Add mobile node i to mobile node k's set of

relationships and vice versa

8: $\mathrm{S}_{\mathrm{k}}=\mathrm{S}_{\mathrm{k}} \cup\{(k, i)\}$

9: $\mathrm{S}_{\mathrm{i}}=\mathrm{S}_{\mathrm{j}} \cup\{(i, k)\}$

10: end

11: end

12: end

13: Use the sets of relationships $X_{k}$ of all the mobile nodes to build a graph $G(A, I)$

14: Set the vertices of the graph $A=I N$ (i.e., vertices are the mobile nodes)

15: Set the edges of the graph $I=U^{N}{ }_{k=1} x_{k}$ (i.e., edges are the mobile nodes' relationships)

16: Identify each group $\mathrm{k}$ of mobile nodes,

IMj $\epsilon A$ where $\cup_{\mathrm{j}} \mathrm{IM}_{\mathrm{j}}=\mathrm{IN}$, which is a maximal complete clique or subgraph in the graph $\mathrm{G}(\mathrm{A}, \mathrm{I})$ obtained by using algorithms such as those in [20].

Mobile node $\mathrm{k}$ is said to have a social relationship with mobile node $\mathrm{i}$ if they meet each other within a required time Tk (i.e., Pki is greater than threshold $\mathrm{w}_{\mathrm{i}}$ and the number of encounters between mobile node $\mathrm{i}$ and mobile node $\mathrm{k}$ is greater than threshold $\mathrm{nth}$ ). Each mobile node would like to reduce the expected packet delivery delay by cooperating with the mobile nodes that it has strong social ties with. Here both $\mathrm{w}_{\mathrm{k}}$ and nth are design parameters which define the "strength" of the social tie. When $\mathrm{w}_{\mathrm{k}}$ increases, the number of mobile nodes in a social group may decrease due to the tighter requirement for encountering. though, the probability to contact those mobile nodes within the required time interval will be higher. On the other hand, when $w_{k}$ decreases, the number of mobile nodes in a social group may increase due to the looser requirement for encountering. However, the chances to contact those mobile nodes within the required time interval will be lower. The threshold nth on the number of encounters is used to ensure that the relationship between a pair of mobile nodes is strong enough. If the value of threshold nth increases, the mobile nodes require stronger relationship to meet the condition. As a result, the number of mobile nodes in a social group may decrease. Conversely, if the value of the threshold nth decreases, the number of mobile nodes in a social group may increase. If the requirement is satisfied, mobile node $\mathrm{k}$ adds 
(k,i)(i.e., its relationship with mobile node $\mathrm{k}$ ) to $\mathrm{X}_{\mathrm{K}}$.

\subsection{COALITIONAL GAME APPROACH}

In a network formation game, the main objective in these game is to study the interactions among a group of players that wish to form a graph[13]. Network formation games can be thought of as a hybrid between coalitional graph games and non-cooperative games.

The reason is that,for forming the network non-cooperative game theory plays an important role.In any network formation game two objectives are of interest: (i)Forming a network graph and(ii)Studying the properties and stability of this graph ,through concepts analogous to those used in canonical coalitional games.

\subsection{BARGAINING MODEL}

To propose a bargaining game formulation to obtain Pareto-optimal solution for the probabilities that mobile nodes will help other mobiles in the same coalition. After the NSA based mobile node grouping is prepared, each group play a coalitional game to obtain a stable coalitional formation. The payoff of every mobile node is a purpose of cost incurred by the mobile node in relaying packets and the delivery delay for packets transmitted to this mobile node from a BS. A continuous-time Markov chain (CTMC) model is formulated to obtain the expected cost and packet delivery delay for each mobile node in the similar coalition. Since the predictable cost and packet delivery delay vary with the probability that each mobile node helps other mobile nodes deliver packets, a game is used to discover the optimal helping probabilities for all the mobile nodes in a coalition .

\section{Conclusion}

We have presented a coalitional game framework for carry-and-forward-based cooperative packet delivery to mobile nodes in a network. The mobile nodes are coherent to form coalitions to maximize their individual payoffs. First, a continuous-time Markov chain model has been developed to obtain the packet delivery delay and the expected cost of mobile nodes for cooperative packet delivery. The packet delivery wait and the predictable cost depend on the probability that each mobile node will help other mobile nodes in the same coalition. Then, a bargaining model game has been formulated to find the optimal helping probabilities for all the mobile nodes. Based on the packet delivery delay and expected cost, a coalitional game has been formulated to model the decision making process of mobile nodes, that is, whether they will cooperatively deliver packets to other mobile nodes or not. A stable coalitional structure (i.e., set of coalitions) has been considered as the solution of this coalitional game. Using the coalitional game model, the performance of cooperative packet delivery has been analyzed in terms of average packet delivery delay. As an extension of the work, the problem of mechanism design can be addressed to enforce truthful packet delivery and prevent the misbehavior of the mobiles nodes under the proposed coalitional game framework.

\section{References}

[1] Khajonpong Akkarajitsakul, Ekram Hossain And Dusit Niyato," Cooperative Packet Delivery In Hybrid Wireless Mobile Networks: A Coalitional Game Approach," IEEE Transactions On Mobile Computing, Vol. 12, No. 5, May 2013.

[2] T. Yamada, R. Shinkuma, and T. Takahashi, "Connectivity and Throughput Enhancement by Inter-Vehicle Packet Relay in Road Vehicle Comm. Systems,” Proc. IEEE Global Telecomm. Conf., pp. 1-5, Nov./Dec. 2006.

[3] Y. Yamao and K. Minato, "Vehicle-Roadside-Vehicle Relay Communication Network Employing Multiple Frequencies and Routing Function," Proc. Sixth Int'1 Symp. Wireless Comm. Systems, pp. 413-417, Sept. 2009.

[4] H. Su and X. Zhang, "Clustering-Based Multichannel MAC Protocols for QoS Provisionings over Vehicular Ad Hoc Networks,'IEEE Trans. Vehicular Technology, vol. 56, no. 6, pp. 3309-3323, Nov. 2007.

[5] W. Saad, Z. Han, M. Debbah, A. Hjørungnes, and T. Basar, "Coalitional Game Theory for Communication Networks: A Tutorial," IEEE Signal Processing Magazine, vol. 26, no. 5, pp. 77-97, Sept. 2009.

[6] D. Niyato, P. Wang, W. Saad, and A. Hjørungnes, "Coalition Formation Games for Bandwidth Sharing in Vehicle-to-Roadside Communications," Proc. IEEE Wireless Comm. and Networking Conf.(WCNC), pp. 1-5, Apr. 2010.

[7] W. Saad, Z. Han, A. Hjørungnes, D. Niyato, and E. Hossain, "Coalition Formation Games for Distributed Cooperation Among Roadside Units in Vehicular Networks," IEEE J. Selected Areas in Comm., vol. 29, no. 1, pp. 48-60, Jan. 2011.

[8] W. Gao, Q. Li, B. Zhao, and G. Cao, "Multicasting in Delay Tolerant Networks: A Social Network Perspective," Proc. ACM MobiHoc, pp. 299-308, May 2009.

[9] E. Daly and M. Haahr, "Network social analysis for Routing in Disconnected Delay-Tolerant MANETs," Proc. ACM MobiHoc,pp. 32-40, Sept. 2007.

[10] S. Pai, T. Roosta, S. Wicker, and S. Sastry, "Using Social Network Theory Towards Development of Wireless Ad Hoc Network Trust," Proc. 21st Int'l Conf. Advanced Information Networking and Applications Workshops, pp. 443-450, May 2007.

[11] S.-L. Hew and L. White, "Cooperative Resource Allocation Games in Shared Networks: Symmetric and Asymmetric Fair Bargaining model Models,” IEEE Trans. Wireless Comm., vol. 7, no. 11, pp. 4166-4175, Nov. 2008

[12] D. Li, Y. Xu, X. Wang, and M. Guizani, "Coalitional Game Theoretic Approach for Secondary Spectrum Access in Cooperative Cognitive Radio Networks,” IEEE Trans. Wireless Comm., vol. 10, no. 3, pp. 844-856, Mar. 2011.

[13] K.R. Apt and A. Witzel, “A Generic Approach to Coalition Formation,” Proc. First Int'l Workshop Computational Social Choice (COMSOC), pp. 21-34, Dec. 2006.

[14] D. Krajzewicz, G. Hertkorn, C. Ro" ssel, and P. Wagner, "SUMO (Simulation of Urban MObility): An Open-Source Traffic Simulation,’Proc. Fourth Middle East Symp. Simulation and Modelling (MESM ’02), pp. 183-187, Sept. 2002. 
[15] R.J. Aumann and J.H. Dr_eze. Cooperative games with coalition structures.International Journal of Game Theory, 3:217 \{237, 1974.

[16] J. Greenberg. Coalition structures. In R.J. Aumann and S. Hart, editors,Handbook of Game Theory with Economic Applications, volume 2 of Handbook of Game Theory with Economic Applications, chapter 37, pages1305\{1337. Elsevier, 1994.

[17] S.S. Yi. Stable coalition structures with externalities. Games and Economic Behavior, 20:201\{237, 1997.

[18] D. Ray and R. Vohra. Equilibrium binding agreements. Journal of Economicn Theory, (73):30\{78, 1997.

[19] A. Bogomolnaia and M. Jackson. The stability of hedonic coalition structures.Games and Economic Behavior, 38(2):201\{230, 2002.

[20] X. Cao, "Preference functions and bargaining model solutions," in Proc. CDC-21, Orlando, FL., Dec. 1982.

[21] T. Spyropoulos, A. Jindal, and K. Psounis, "An Analytical Study of Fundamental Mobility Properties for Encounter-Based Protocols," Int'l J. Autonomous and Adaptive Comm. Systems, vol. 1, no. 1, pp. 4-40, July 2008

[22] W. Gao and G. Cao, "On Exploiting Transient Contact Patterns for Data Forwarding in Delay Tolerant Networks," Proc. IEEE 18th Int'l Conf. Network Protocols (ICNP), pp. 193-202, Oct. 2010

[23] Z. Xinjuan and X. Bo, "A Traffic Resource Diffusion Scheme in Vehicular Networks," Proc. Int'l Forum on InformationTechnology and Applications, pp. 318-321, May 2009.

[24] R. Groenevelt, P. Nain, and G. Koole, "The Message Delay in Mobile Ad Hoc Networks," Elsevier J. Performance Evaluation, vol. 62, nos. 1-4, pp. 210-228, Oct. 2005. 